\title{
Towards Autonomous Synthesis of Building Substation Control Loops
}

\author{
Wolfgang Birk ${ }^{1}$, Khalid Atta ${ }^{1}$
}

\begin{abstract}
The main contribution of this paper is to study the feasibility of an automated approach to the assessment and synthesis of control loops in substations of a district heating and cooling system. The low-level controllers in substation are usually PID-type controllers and can not easily be changed. Moreover, the tuning of these controllers is often done in an ad-hoc manner and remains in the initial tuning despite changes in the substation over their operational life. Consequently, insufficient control performance due to false tuning can be misinterpreted as a hardware issue and lead to unnecessary replacement of parts.

In order to solve this problem, the paper proposes an approach to assess the control loop performance based on logged data from substations, and to subsequently tune the current substation controllers, when no hardware issues are detected. The approach is applied to a well-known building substation model in simulation, which indicates that the approach is feasible.

Analysis of acquired data from a number of substations in the DHC system of Luleå Energi $\mathrm{AB}$, provides evidence for the problem and how the approach can be applied in its future implementation. Moreover, a number of challenges and future work are indicated.
\end{abstract}

Index Terms-district heating, substation control, performance assessment, auto-tuning, temperature control, tap water control

\section{INTRODUCTION}

District heating and cooling systems (DHC) are widely spread and supply buildings with heat and cold for space heating and cooling as well as tap water heating. The centralized production of heat and cold has numerous advantages and challenges which are thoroughly discussed in [1]. One of the largest advantages is that heat production is centralized in combined heat and power plant, which are highly automated and optimized in their operation.

In a DHC system the consumers are connected to the supply network through substations which use the supplied energy to locally extract heat and cold using heat exchangers. Usually, the heat exchangers are actuated on both primary and secondary side to provide water flows with a desired temperature. For this end control loops are in place which make use of measurements from both sides. For the case of tap water, the flow through the primary side of the heat

\footnotetext{
${ }^{1}$ Wolfgang Birk, and Khalid Atta are with Control Engineering Group, Department of Computer Science Electrical and Space Engineering, Luleå University of Technology, Luleå, Sweden. \{wolfgang.birk; khalid.atta\} eltu.se
}

exchanger is adjusted to achieve a desired water temperature, while the desired flow is achieved through measurements and actuation on the secondary side.

The control loop performance now depends on a number of aspects. First of all, the flow control valve on the primary side need to be correctly dimensioned to achieve proper heating of the second side water flow. Secondly, the temperature control loop need to be well designed to attain the desired control objectives in a predefined timely manner and to manage load disturbances. In this context load disturbances are fluctuations in the use of tap water and the temperature variations on the primary side.

A typical problem in this context is the proper tuning of the control loops for the tap water. In many cases, PID controllers are used to maintain a constant tap water temperature which is tuned during installation of the substation. Later tuning of the loop is rarely done and mostly based on ad-hoc tuning which does not rely on an analysis of the substation behaviour. As a result, the substation is not optimally tuned and undesired dynamic behaviour of the closed loop system may occur.

In the process industry, auto-tuning of controllers is a natural ingrediens in control systems nowadays and the research area is mature, see [2] for a comprehensive summary. Nevertheless, the control engineering community is still developing improved methods and tools and to the knowledge of the authors, such tools are usually not available or used in DHC community. Despite the task of auto-tuning determining a controller from a process model is also a mature topic in the area of process control. A good summary of efficient methods for the design and tuning of PID controllers is given in [3].

Although there is a plethora of methods, the DHC community has a lack of tailored methodologies and tools that support engineers in optimizing substations in buildings in an efficient and systematic manner.

The novelty and contribution of this paper lies in proposing a tool for model-based automated controller synthesis of substation control loops. The approach can make use of two modeling principles which render a linear dynamic process model in the end. The first principle uses dynamic physical models for a substation which are parameterized using either gray box modeling from experimental process data or from a component data sheets, while the second depends on black box modeling from experiments alone. The approach will support the automation engineer to determine the current 


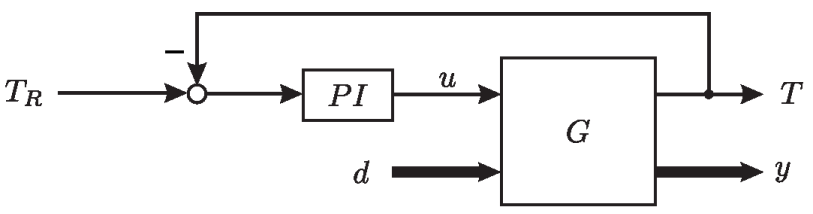

Fig. 1. Principle sketch of the control system setup that is used for the analysis of the substation control loop.

performance characteristics of the substation control system and to determine controller parameters for PID controllers used in temperature control for the tap water.

The paper is laid out as follows. First, a more thorough problem definition is given followed by an overview of the proposed approach. Thereafter the performance assessment and automated controller synthesis methods are described and applied in a case study. The case study is split into a simulation study and analysis of operational data from substations in the DHC system of Luleå Energy AB.

\section{PROBlem DESCRIPTION AND APPROACH}

It has already been stated that the temperature control for the tap water uses a heat exchanger which is actuated on the primary side to adjust the temperature on the secondary side. Usually the control loop is designed as a single-input singleoutput system, where the secondary side flow control can be understood as a load disturbance. Moreover, temperature fluctuations on the primary side need to be considered as disturbances as well. Those disturbances can be measured but are either the result of an actuation in an interconnected control loop or are exogenous.

A principle sketch of this setup is given in Fig. 1. There, the temperature control loop has $T$ as the measured output, $T_{R}$ as the set point and $u$ as the control signal that actuates the flow control valve on the primary side. Additionally, all disturbances are lumped into the vector $d$. The remaining measurable signals are combined into the vector $y$. As such the complete system can be interpreted as a multivariable feedback system. Note that certain elements in $y$ and $d$ can be part of other feedback loops.

Traditionally, the PID controller synthesis is based on tuning methods which use information on the components of the closed loop system, like e.g. heat exchanger characteristics and flow control valve characteristics. First principle models or black box models of the system are usually not used in practice, as there has been limited access to operational data and apparent limitations to conduct experiments on the system.

The aim is now to determine a methodology which combines gray-box and black box modelling on the system in order to automatically synthesize a controller for the tap water temperature control, if the performance of the closed loop system is insufficient.
For sake of simplicity all signals in $d$ are assumed to be exogenous and thereby no additional interconnected loops are present which relate $d$ with $y$. Furthermore, it is assumed that the non-linear system can be approximated sufficiently well by a linearized model for a certain operating point, which yields the following system representation in the Laplace domain:

$$
\begin{aligned}
{\left[\begin{array}{l}
T \\
y
\end{array}\right] } & =\left[\begin{array}{ll}
G_{T u}(s) & G_{T d}(s) \\
G_{y u}(s) & G_{y d}(s)
\end{array}\right] \cdot\left[\begin{array}{l}
u \\
d
\end{array}\right] \\
u & =P I(s)\left(T_{R}-T\right)
\end{aligned}
$$

Here, $G_{\bullet}(s)$ and $P I(s)$ denote the transfer function matrices between the signals or signal vectors.

For an automatic assessment and synthesis of a temperature controller, the following approach is now proposed:

1) Derive a specification of desired performance of the temperature control loop

2) Analysis of the current temperature control loop performance

3) Update or derivation of $G_{T u}(s)$

4) Automatic synthesis of $P I(s)$ from the specification and $G_{T u}(s)$

5) Commissioning of the new controller $P I(s)$

6) Restart at step 1 or 2.

Clearly, the approach largely depends on the availability of a performance specification and access to the substation. The access to the substation can be either in an direct online access as it is provided by system from NODA [4], Abelko [5], or through data logging which is conducted by engineers at the individual substation. In the context of this study it is assumed that the specification and data is available. Moreover, it is assumed that the specification can be mapped into desired closed loop transfer functions of the temperature control loop, which will be denoted $\hat{P}_{T}(s)$ and $\hat{P}_{S}(s)$ for the complementary sensitivity transfer function and sensitivity transfer function, respectively.

\section{LOOP PERFORMANCE MONITORING}

Before a controller is re-designed it is essential to determine if the control is not well-performing. Loop performance assessment is an active research area in the process control community and aims at monitoring the performance of a single control loop or many control loops at the same time.

The first research results can be traced back to the work by Åström [6] and DeVries and Wu[7]. In both cases, research was conducted to evaluate the performance of control loops in a paper mill. Later, Harris [8] developed a performance assessment technique only using data from process operation for univariate control loops both in SISO and MIMO system. The evaluation was done based on the the variance in the control system, where minimum variance control is a natural choice [9]. 
The key concept in Harris work is to identify if the loop performance is poor due to the current controller design or due to external disturbances. The procedure derived in his work is proven to be able to diagnose the cause of the performance deficiency.

From a practical perspective, loop performance monitoring (or simply loop monitoring) is a crucial and important part in any industrial control system. Nowadays, many commercial products are available to analyse and diagnose the performance of the loops on a local level (i.e. considering the performance of individual control loops) or plant wide level (i.e. considering the whole plant and detect if there are plant wide disturbances that affect different parts).

A recently conducted survey showed that this subject has been a major research topic for the last 25 years and will stay important in the future due to its important role and benefits in the industry, more details can be found in [10].

In the scope of this paper, the loop performance monitoring task is assumed to be solved. In an online tool, the loop performance monitoring has to run in parallel with the current control system, while in an offline tool the loop performance is usually previously observed by an engineer or operator during operation. In the latter case, data will be acquired and assessed. The assessment can be either done by the engineer manually or employing the above tools.

In the context of this study it can be concluded that the current state of the art is sufficient to perform needed assessment and detect the source of performance deficiency.

\section{Autonomous Controller Synthesis}

When the assessment of the closed loop system indicates that the performance is insufficient, three cases have to be considered: (i) the hardware has changed and the controller need to be adapted or re-tuned, (iii) operating conditions are alterned and require adaptation or retuning of the controller, (ii) the hardware is defect or inappropriate need to be exchanged. In the latter case, a subsequent controller redesign is needed. Thus, in all three cases a new controller need to be found which replaces the current one, for which a model of the current substation is needed.

\section{A. Model update}

The model $G_{T u}(s)$ which is used for the controller synthesis need to be updated to fit the current operational and hardware characteristics. There are two main tracks that should be considered:

- Gray-box: Determining the parameters of a first principle model, which is subsequently linearized and the model $G_{T u}(s)$ is extracted.

- Black-box: Determining the model $G_{T u}(s)$ directly.

For both tracks data is needed, which is why the black-box approach is favored over the gray-box in the scope of this study. Data is then used to determine the model parameters in a pre-determined model structure. Since it is difficult to take the closed loop system out of operation, the estimation of parameters has to be done in closed loop. Resultingly, the effects of the closed loop on the estimation of the parameters need to be considered [11].

Usually, the practical approach is to try direct system identification of $G_{T u}(s)$ using logged data of $u$ and $T$ from either operation or experiments with the closed loop. At this point one needs to be careful where the excitation signal originates from, $d$ or $T_{R}$. In the first case, the excitation signal is $d$, then it can be shown that the transfer function which is derived from $T$ and $u$ is

$$
\frac{T(s)}{u(s)}=-\frac{1}{P I(s)}
$$

This means that the acquired signals from the experiment reflect the controller and not the system. In the second case, the excitation signal is $T_{R}$, which actually proves that the transfer function derived from $T$ and $u$ is $G_{T u}(s)$. While this is encouraging, it is important to make sure that the excitation originates from $T_{R}$ and not from $d$.

As an alternative, the closed loop system can be excited through exogenous signals in the vector $d$ or $T_{R}$, and the closed loop transfer function $P_{T}(s)$ or $P_{S}(s)$ can be derived from direct system identification. Since the controller transfer function $P I(s)$ is known, the $G_{T u}$ can be calculated from the previously derived $P_{T}(s)$ or $P_{S}(s)$.

We will now assume that only one element in $d$ is used to excite the system, which means that all transfer functions are single-input and single-output. Now it is straightforward to extract the system model $G_{T u}$ from either $P_{T}(s)$ or $P_{S}(s)$ as follows:

$$
\begin{array}{r}
G_{T u}(s)=\frac{P_{T}(s)}{P I(s)-P_{T}(s) P I(s)} \\
G_{T u}(s)=\frac{G_{T d}(s)-P_{S}(s)}{P_{S}(s) P I(s)}
\end{array}
$$

Depending on the design of the substation the models for $G_{T u}(s)$ and $G_{T d}$ will differ. Nevertheless, in many cases simple transfer functions are sufficient to describe the dynamic behaviour of a substation. From a synthesis perspective of a PI controller, the following model structures are preferred

$$
\begin{aligned}
G_{T u}(s) & =\frac{k}{\tau s+1} \\
G_{T d}(s) & =k_{d} \\
P I(s) & =k_{p}\left(1+\frac{1}{\tau_{i} s}\right)
\end{aligned}
$$

Consequently, the closed loop transfer functions will be of order 2 with either one or two zeros, for $P_{T}(s)$ or $P_{S}(s)$, respectively. In case that the complexity of the model $G_{T u}$ is 
increased with an additional time constant or time delay, then the order of the closed loop transfer functions is increased accordingly.

For the assumed model structures in (5), (6) and (7), the structure of the closed transfer functions are

$$
\begin{aligned}
& P_{T}(s)=\frac{k \cdot k_{p}\left(\tau_{i} s+1\right)}{(\tau s+1) \tau_{i} s+k \cdot k_{p}\left(\tau_{i} s+1\right)} \\
& P_{S}(s)=\frac{k_{d}(\tau s+1) \tau_{i} s}{(\tau s+1) \tau_{i} s+k \cdot k_{p}\left(\tau_{i} s+1\right)}
\end{aligned}
$$

Similarly, the closed loop transfer functions can be derived for a second order $G_{T u}(s)$, where there is a slow mode and a fast mode. Then the resulting transfer functions for $P_{T}(s)$ and $P_{S}(s)$ will have order three. Now that there are three different options to determine the model $G_{T u}(s)$ and depending on the characteristics of the data, the most appropriate one is chosen.

\section{B. Controller synthesis}

Again, the aim is to determine the parameters of a PI Controller from a known $G_{T u}(s)$. There a numerous wellproven synthesis concept, of which the so-called $\lambda$-tuning is an established standard in the Swedish pulp and paper industry. A summary on available design tools can be found in [3]

The $\lambda$-tuned controller is based on the inverse of the model and is a straight forward way to achieve a desired closed loop bandwidth. The controller is simply used to cancel the poles of the process by correctly adjusting the controller zeros. Thereby the controller need to have equally many zeros as there are poles in the process model. By conducting a model order reduction to the dominant dynamics of appropriate order a PI or PID controller can be found as

$$
C(s)=\frac{1}{G_{T u}(s)} \frac{1}{\lambda s}
$$

The advantage of this method is the simplicity and the sensitivity of the closed loop is always less than two for an exact process model of the plant, which means that closed loop system fulfills certain robustness properties towards model uncertainty.

In this study, $G_{T u}(s)$ will have at most order two, which means that an order reduction is performed in case that $G_{T u}(s)$ is of order two and a PI controller should be synthesized. This is achieved by neglecting the mode with less contribution to the overall dynamics.

Thereafter $\lambda$ is chosen to reflect the bandwidth from the specification $\hat{P}_{T}(s)$.

\section{Simulation StUdy}

In the simulation study we will now focus on the steps 3 to step 5. Most importantly, the feasibility of these steps shall be evaluated. A simulation example will be used to demonstrate the steps model update, controller synthesis and evaluation of a small house heating substation.

The simulation model is based on the physical thermodynamic model of a detached house connected to a district heating network. The model was presented in [12] and validated in [13]. The parameters of the simulation was the same as House A in [14].

The substation is controlled using a PI controller with transfer function

$$
P I(s)=\frac{1}{150}\left(1+\frac{1}{150 s}\right) .
$$

Usually, sensors contain a filter to attenuate the noise effect and to provide more smooth data to the controller. The cutoff frequency of the sensor mainly depends on the type of the transducer, the signal quality, and the fastness of the controller. Here, the low pas filter was designed to be at least six times faster than the control loop and has the transfer function,

$$
F(s)=\frac{1}{s+1} .
$$

The controller set point $T_{R}$, as shown in Fig. 1, is provided through a look up table that depends on the outdoor temperature. In order to excite the system, a square perturbation signal with amplitude $2^{\circ} \mathrm{C}$ and frequency $\frac{1}{600} \mathrm{~Hz}$ was superimposed on the outdoor temperature. Fig. 2 shows the results of the perturbed system. Note that the simulation was done under a time varying weather condition and supply conditions. Thus it can be noted that the average set point (the blue curve in the top plot) is decreasing with time.

It is clear that the current PI controller does not track the requested set-point properly. Thus, in order to examine the behavior of the PI, the closed loop transfer function $P_{T}(s)$ was identified using Matlab's system identification toolbox.

$$
P_{T}(s)=\frac{0.4681 s+0.0004684}{s^{3}+76.65 s^{2}+0.5448 s+0.0004684}
$$

Note that the order of the identified system was selected to be of order three, with at least one zero. It is possible to have higher order systems that provide a better fitting to the provided data, but a higher order would not be practical nor realistic based on the previous discussion. Based on the known $P I(s)$, the plant transfer function was found as

$$
G_{T u}(s)=\frac{70.22 s+70.22}{s^{2}+76.65 s+0.511}
$$

Using the estimated plant transfer function, a synthesis of the PI controller was conducted and resulted in 

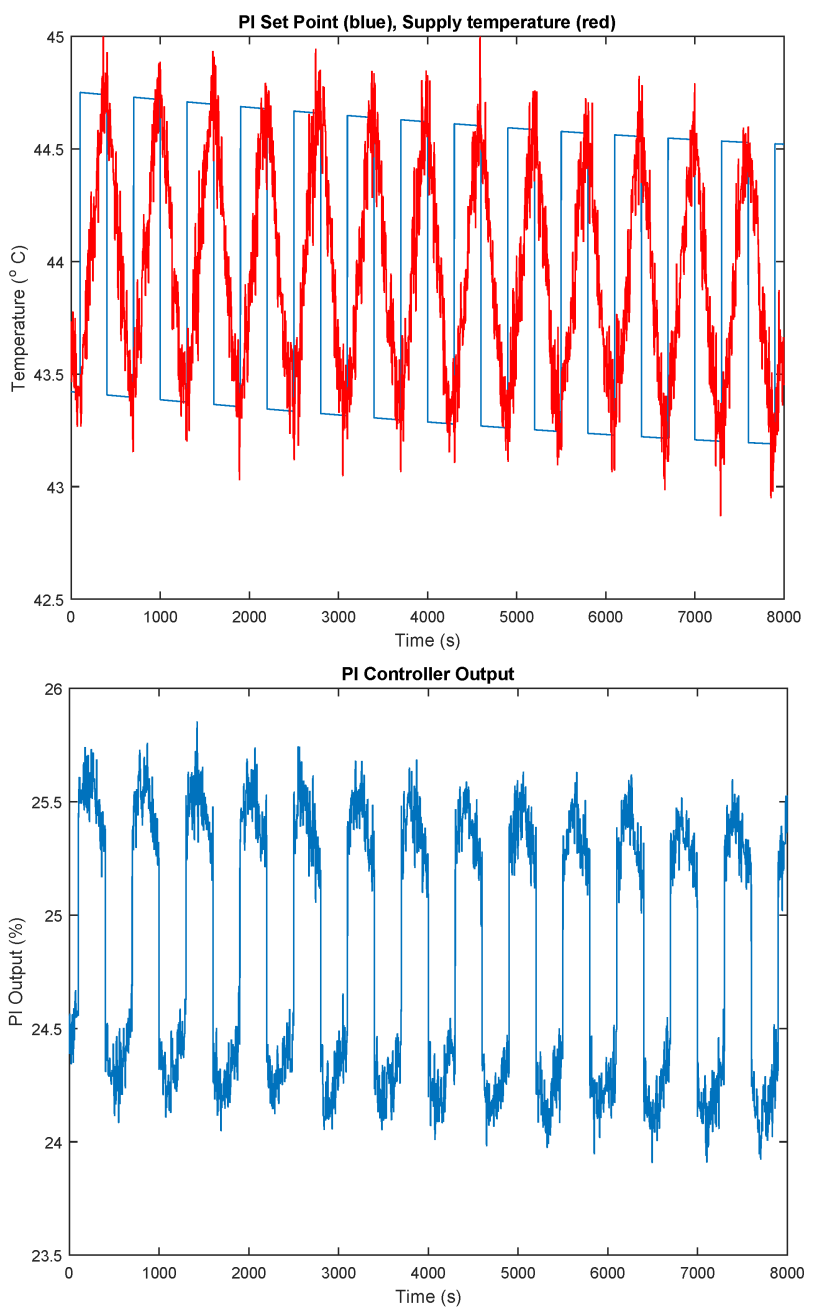

Fig. 2. Simulation of a small substation. Top: set point values for the temperature (Blue), temperature measurement(Red). Bottom: PI controller output \%.

$$
P I^{*}(s)=0.26\left(1+\frac{1}{50 s}\right) .
$$

Fig. V shows a comparison between the response of the original and the autonomously synthesized system. The blue curve shows the set point, while the red represents the response of the original system and the black represents the tuned system. It is clear that the autonomously synthesized system provides a better tracking of the set point, but with some overshoot that can be removed with a less aggressive system specification

\section{REAL LIFE CASES}

For a preliminary analysis of the feasibility of this approach, operational data has been acquired from three substations in different building sites. For each of these sites the primary side flow control valve was acquired and the secondary side temperature of the tap water. The control

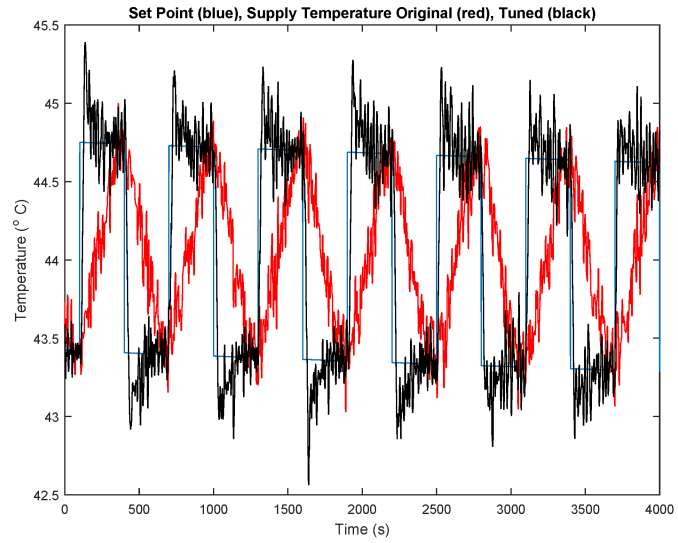

Fig. 3. Comparison between the original and the preliminary tuned PI controllers. To the left: set point values for the temperature (Blue), temperature measurement original (red), temperature measurement tuned (black). To the right: flow control valve opening in $\%$.

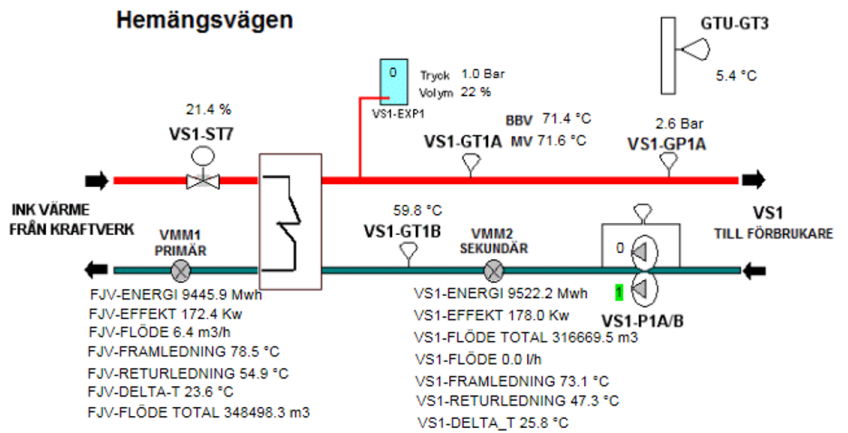

Fig. 4. Screenshot of the operator interface for the building site at Hemängsvägen. The flow control valve on the primary side is denoted VS1ST7, and the temperature sensor is denoted VS1-GT1A. Momentary values for the valve opening and temperature are depicted close to the component.

performance and control action in closed loop will be analyzed and it will be judged if the proposed approach can be applied.

\section{A. Building site: Hemängsvägen}

The buildings at Hemängsvägen do have a substation which converts the supplied heat to a local heating system for both space heating and tap water heating. In other words, a local district heating system is created with its own supply temperature to the individual buildings. Moreover, the supply temperature on the secondary side is higher than for usual tap water during winter time.

In Fig. 4, an example for a substation is depicted. The typical structure of the substation as well as the placement of the temperature sensor and flow control valve can be seen. For this specific case, the substation is used for both space heating and tap water production. As a result the secondary water temperature is relatively high and not fixed. For control of the temperature a PI controller in a one degree of freedom control architecture with proportional gain $k_{P}=50$ and an integration time of $T_{I}=180 \mathrm{~s}$ is used. 

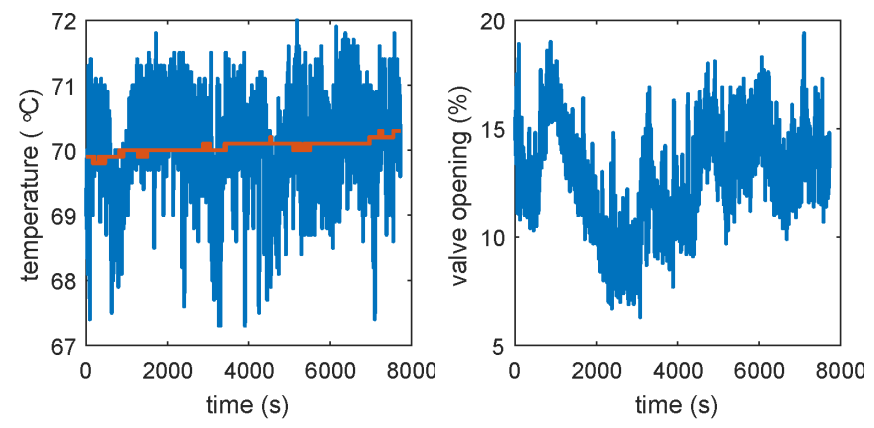

Fig. 5. Example for a data sequence for the substation at Hemängsvägen. To the left: temperature measurement VS1-GT1A (Blue), set point values for the temperature (orange). To the right: flow control valve VS1-ST7 opening in $\%$.

In Fig. 5, a data sequence for the temperature measurement, its associated set point value and the flow control valve opening are displayed. The data was acquired with a sample rate of $1 \mathrm{~s}$. Here it can be seen that the measurement values are fluctuating around the set point, and from inspection it seems that the mean of the measurement is close to the desired set point value. An evaluation of the data reveals that the mean error is $0.0059{ }^{\circ} \mathrm{C}$ and the standard deviation is $0.7330^{\circ} \mathrm{C}$. It can also be seen that the controller is active and attenuates deviations from the set point and essentially succeeds in the task. Moreover, the temperature measurement reveals a relatively large amount of noise, which is also reflected in the quite large instantaneous control actions.

In this case, it seems that excitation of the system through the set point $T_{R}$ is feasible to acquire the needed data for system identification and subsequent controller synthesis.

\section{B. Building site: Traktorvägen}

In contrast to the building site at Hemängsvägen, the tap water at Traktorvägen is directly extracted from the substation in accordance with Fig. 1. In order to reduce the risk for a legionella bacteria spread the temperature of the tap water has a minimum level of $56{ }^{\circ} \mathrm{C}$ and will be kept constant with $T_{R}=57{ }^{\circ} \mathrm{C}$. Obviously, this creates the issue that the closed loop system is not naturally excited by the set point $T_{R}$ and system identification of $G_{T u}(s)$ from the acquired data is not possible. As stated above the identification would render a model of the inverse of the controller and not of the system itself.

For a time period of 80000 seconds a time series of the temperature $T_{R}$ and the valve opening is acquired with a sample rate of one second and depicted in Fig. 6. There it can be seen that the controller achieves quite good performance with a mean error of $0.0017{ }^{\circ} \mathrm{C}$ and a standard deviation of $0.8113{ }^{\circ} \mathrm{C}$. The control actions are also moderate and do seem to attenuate the disturbances swiftly.

A closer look in the time range between 50000 and 70000 seconds reveals that the control valve seems to oscillate
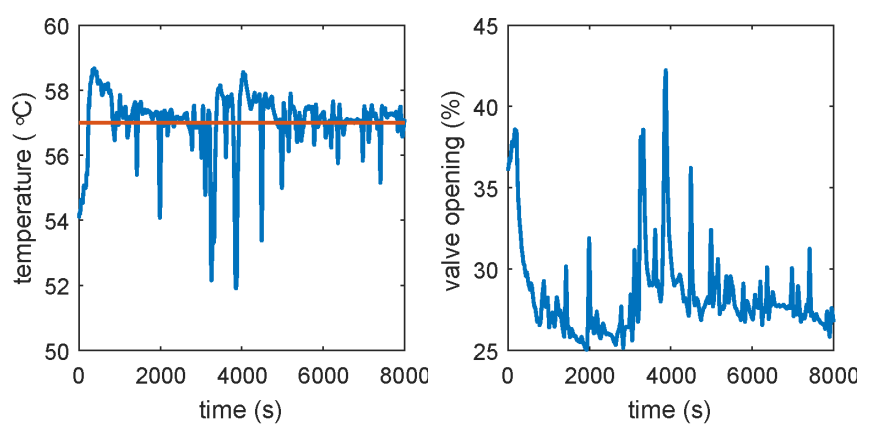

Fig. 6. Example for a data sequence for the substation at Traktorvägen. To the left: temperature measurement $T$ (Blue), set point values for the temperature $T_{R}$ (orange). To the right: flow control valve opening $u$.

around a relatively low opening. Similarly, the temperature $T$ is also oscillating. This oscillation seems to be unmotivated as there are no apparent load disturbance peaks during earlier parts of the time series. It is likely that the oscillations stems from a limit cycle in the control system, which could be caused by quantization or stiction phenomena in the valve. Such behaviour will most likely effect the useful life of the valve and lead to increased maintenance costs.

A deeper analysis of the causes for this behaviour is needed in order to capture and mitigate such behaviour.

Nevertheless, in this case it is necessary to either excite the system through experiments with load disturbances or set point variations, otherwise a gray-box approach is needed to acquire the model parameters.

\section{Building site: Porsögården}

At the Porsögården site, the engineers are suspecting a malfunctioning valve or an oversized valve. The building site has the disadvantage that the substation is not equipped with a control system that allows the acquisition of measurement data for the temperature directly. Instead a temporary measurement device need to be attached to the supply temperature line which measures the temperature indirectly through contact with the metal shell of the pipe. This leads to a bias in the measurement and to a smoothing of the temperature curve. The smoothing can be understood as a type of low-pass filtering.

In Fig. 7, the acquired data is displayed. First of all, the bias can be easily seen to be approximately $5{ }^{\circ} \mathrm{C}$, as the set point is also $T_{R}=57{ }^{\circ} \mathrm{C}$. Moreover, there are few load fluctuations in the system and the temperature oscillates around the set point. In the control actions it can be seen that the valve is opened at the lower end of its scale and that there seem to be quantization phenomena present in the valve. A closer look reveals that the valve mainly operates on four to five discrete opening levels. This type of quantization can lead to limit cycles which are most likely present in this system. 

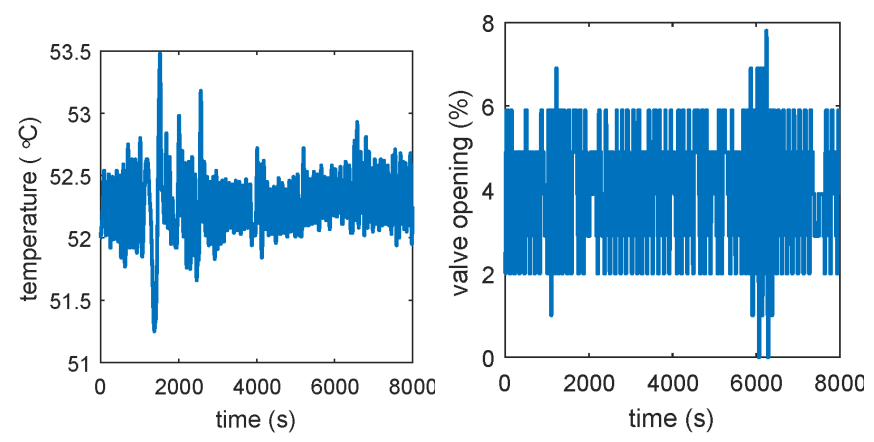

Fig. 7. Example for a data sequence for the substation at Porsögården. To the left: temperature measurement $T$ (Blue), set point values for the temperature $T_{R}$ (orange). To the right: flow control valve opening $u$.

Moreover, the valve does only operate at a certain low level all the time which means that a smaller valve would be more appropriate for this kind of substation. In this case, there are several options for the engineer. Firstly, the valve could be replaced by a smaller valve and then the limit cycle could disappear and a more smooth operation can be expected. Secondly, the engineer could perform a re-scaling of the control system's measurement units and thereby operate with a better resolution on the same equipment. From the current information it is difficult to draw any conclusion on the performance of the control system itself besides that a constant level i maintained, which could have a remaining constant control error.

\section{CONCLUSION}

This study proposes an approach for autonomous performance assessment and control synthesis of building substations. The approach is a combination of established theories and methods and could be integrated into a tool that is continuously monitoring the performance of a building substation. If the performance deviates from the desired characteristics, the tool can initiate a synthesis step for an alternative controller.

In the short term and to understand potential limitations in the approach, the tool can be implemented as an offline tool, where engineers can use the tool to analyse substations, get decision support on either component replacement or redesign of the currently used controller. The synthesis of an alternative controller is done automatically. The engineers task is then the commissioning of the new controller or controller parameters.

The paper has provided details on the approach and how it can be realized. A simulation study using a well known building substation model has been used to establish the feasibility. Moreover, real life substation data is acquired and analysed to get an understanding of the feasibility of the approach in a real-life setting, as well as an understanding of the challenges which further need to be addressed.

It is the belief of the authors that the approach is feasible and to conduct a field test with an implemented offline tool that assists an engineer in their work.

\section{ACKNOWLEDGEMENT}

The authors want to thank Luleå Energi $A B$ for their support and providing access to substations. The support provided by Håkan Sundberg and Hans Englund is here specially recognized. Funding provided by the European Commission's Horizon 2020 research programme under grant number 649796 (OPTi project, www.opti2020.eu) is hereby gratefully acknowledged.

\section{REFERENCES}

[1] S. Frederiksen and S. Werner, District heating and cooling. Studentlitteratur, 2013.

[2] T. Hägglund, "Autotuning," Encyclopedia of Systems and Control, pp. 50-55, 2015.

[3] P. Cominos and N. Munro, "Pid controllers: recent tuning methods and design to specification," IEE Proceedings-Control Theory and Applications, vol. 149, no. 1, pp. 46-53, 2002.

[4] NODA, "Index - NODA Intelligent Systems," 2015. [Online]. Available: https://www.noda.se/en/main

[5] Abelko, "Abelko Innovation - Automation, energy effectivity and web solutions," 2015. [Online]. Available: http://www.abelko.se/default.aspx

[6] K. J. Åström, "Computer control of a paper machine: an application of linear stochastic control theory," IBM Journal of research and development, vol. 11, no. 4, pp. 389-405, 1967.

[7] W. DeVries and S. Wu, "Evaluation of process control effectiveness and diagnosis of variation in paper basis weight via multivariate timeseries analysis," IEEE Transactions on Automatic Control, vol. 23, no. 4, pp. 702-708, 1978.

[8] T. J. Harris, "Assessment of control loop performance," The Canadian Journal of Chemical Engineering, vol. 67, no. 5, pp. 856-861, 1989.

[9] B. Huang and R. Kadali, "Control loop performance assessment: Conventional approach," in Dynamic Modeling, Predictive Control and Performance Monitoring. Springer, 2008, pp. 145-155.

[10] M. Bauer, A. Horch, L. Xie, M. Jelali, and N. Thornhill, "The current state of control loop performance monitoring-a survey of application in industry," Journal of Process Control, vol. 38, pp. 1-10, 2016.

[11] U. Forssell and L. Ljung, "Closed-loop identification revisited," Automatica, vol. 35, no. 7, pp. 1215-1241, 1999.

[12] J. Gustafsson, J. Delsing, and J. Van Deventer, "Thermodynamic simulation of a detached house with district heating subcentral," in Systems Conference, 2008 2nd Annual IEEE. IEEE, 2008, pp. 1-8.

[13] J. Gustafsson, J. Delsing, and J. van Deventer, "Validation of a district heating substation model using a wireless sensor network approach," in IDEA Annual Conference \& Trade Show, 2008.

[14] —, "Improved district heating substation efficiency with a new control strategy," Applied Energy, vol. 87, no. 6, pp. 1996-2004, 2010. 